\title{
Regularities in species niches reveal the World's climatic regions
}

\author{
Joaquín Calatayud,* Magnus Neuman, Alexis Rojas, Anton Eriksson, and Martin Rosvall \\ Integrated Science Lab, Department of Physics, Umeå University.
}

(Dated: November 21, 2019)

\begin{abstract}
Although classifications of the Earth's climates date back to the ancient Greeks, the climatic regions shaping the distribution of animals remain poorly resolved. Here we present a classification of global climates based on regularities in realised niches of 3657 amphibians, 7204 reptiles, 10684 birds and 4574 mammals. We found 16 main climatic regions that are mostly consistent across groups and previous plant expert-based classifications, confirming the existence of major climatic restrictions for life. The results also suggest that differences among groups likely relate to their particular adaptations and dispersal capabilities. We further show how the integration of species niche classifications with geographical information provides valuable information on potential mechanisms shaping the climatic regions. Our climate classification has applications in several disciplines, including conservation planning and ecological and evolutionary studies.
\end{abstract}

\section{INTRODUCTION}

6 Climate governs the basis for life on Earth. Besides 7 historical contingencies and geographical barriers, abiotic 8 conditions determine species ranges [1-3] and derived di9 versity patterns $[4,5]$. On a global scale, distinctive cli10 matic regimes impose generalised restrictions, leading to 11 the formation of species pools adapted to them and ul12 timately to the generation of biomes [6]. Identifying the 13 boundaries of these climate regimes is, therefore, a fun14 damental challenge to understand how life organizes on ${ }_{15}$ Earth.

${ }_{16}$ Already Pythagoras proposed a classification of cli17 mate regimes of the known world in the sixth century ${ }_{18} \mathrm{BC}$ [7]. However, it was not until the 19th century when 19 geographers laid the foundations for such classifications 20 [8]. By that time, researchers noticed the close rela${ }_{21}$ tionship between the distribution of various life forms, 22 especially vegetation types, and climate [8]. For in23 stance, Köppen built his long-standing climate classifi24 cation from pioneer plant classifications, assuming that 25 vegetation forms carry information about climatic con26 ditions $[9,10]$. This assumption has received consider27 able support [11], and the Köppen classification system 28 is widely used nowadays as the standard classification 29 of climates in a range of disciplines, including climatolso ogy [12], geography [13], conservation planning [14], and 31 ecology [15]. However, the fact that plant species are 32 good indicators of general climatic conditions does not ${ }_{33}$ necessarily imply that such conditions restrict the dis34 tribution of other organisms in the same manner. If 35 different taxa have different climatic adaptations, the 36 boundaries defining climate types will vary among them. ${ }_{37}$ Following Thornthwaite [10], the "truly active factors" 38 describing a climate type may vary among organisms. 39 Thus, while Köppen's climate classification can indicate 40 the active climatic factors for plants, it remains unknown ${ }_{41}$ whether they are also appropriate for other organisms.

\footnotetext{
* Corresponding author: j.calatayud.ortega@gmail.com
}

${ }_{42}$ Despite several attempts to refine or propose alternative ${ }_{43}$ climatic regions [16-19], quantitative studies defining cli44 matic regions for other organisms are still lacking.

The current information on species distributions and 46 global climatic variables, together with recent advances ${ }_{47}$ in niche modelling and classification techniques provide ${ }_{48}$ an unprecedented opportunity to identify the climatic 49 boundaries shaping the distribution of faunas and flo50 ras across the globe. The last decades have witnessed 51 a tremendous collective effort to record occurrences of 52 a large number of species [20], which has resulted in 53 comprehensive datasets with the distributional ranges of ${ }_{54}$ several groups [21-23]. Also, data on climatic variables 55 at a global scale have been developed at high spatial 56 resolutions $[24,25]$. This information allows to charac57 terise the realised climatic niches of diverse species and ${ }_{58}$ to find regularities among them. For example, project59 ing these realised climate niches into a climatic space [26] 60 should, if climatic boundaries exist, reveal co-occurring ${ }_{61}$ groups of species across particular portions of the cli${ }_{62}$ matic space. Thus, identifying these portions, or niche ${ }_{63}$ domains, should uncover the main climatic boundaries 64 shaping the organization of life (Fig. 1).

${ }_{65}$ Besides climate shaping niche domains, dispersal bar66 riers and historical contingencies may also influence their 67 shape $[3,27,28]$. Therefore, similar climates may have ${ }_{68}$ different effects across geographic regions [29]. For in69 stance, while a given climate may lead to specific species 70 pools in some parts of the Earth, the same climate in 71 other parts of the Earth may not hold specific species 72 pools. Such lack of specific species can occur, for ex73 ample, because the required adaptations have not ap${ }_{74}$ peared [30], the adapted species have been not able to 75 disperse [31], or the area is too small to hold large species 76 pools [32]. Thus, studying the signature of these histor77 ical and geographical processes, the geographical signal 78 for short, in niche domains can provide valuable informa79 tion about the potential mechanisms behind them and 80 their associated climatic regions.

${ }_{81}$ Here we explore the global climate regions of ${ }_{82}$ Tetrapoda by characterising the climatic niche domains 83 of amphibians, birds, mammals and reptiles. Tetrapoda 
1) Raw data

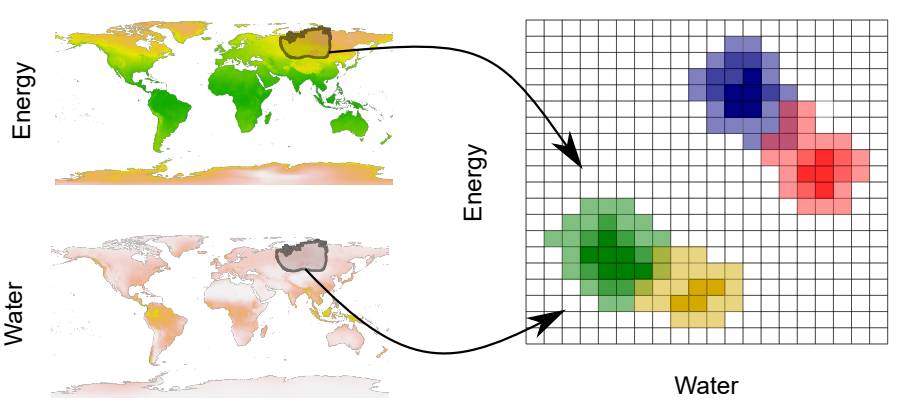

2) Species niches

Water
3) Network modules

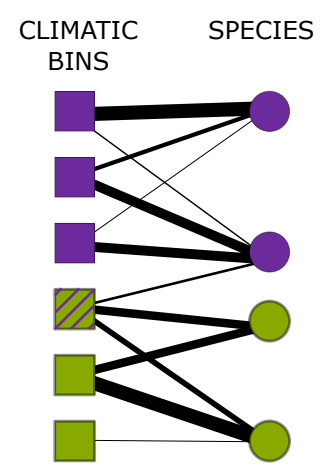

4) Niche domains and climatic regions
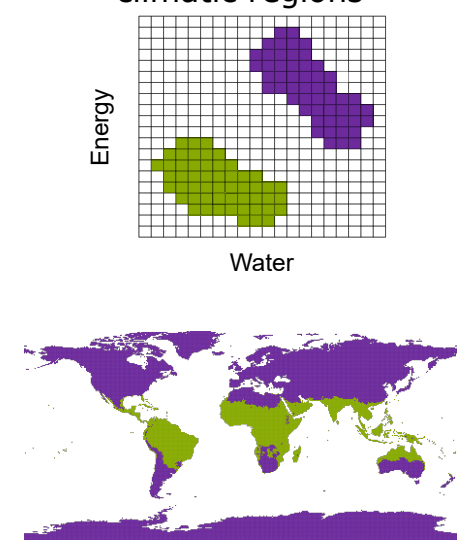

FIG. 1: Workflow to identify niche domains and climatic regions. Using the climatic conditions a given species experiences within its range (1), we project the species's niche into a climatic space discretised in an optimal number of bins (Appendix S1) (2). We translate the binned data into a weighted bipartite network, where climatic bins and species form the nodes and the probabilities of finding the species in the bins form the weighted links (3). Using a network community detection algorithm, we identify domains of the climatic space with similar species (4, upper). The climatic conditions defining these domains delineate the corresponding climatic regions of the Earth (4, lower). The striped climatic bin is linked to species classified in both climatic domains and, therefore, it represents a diffuse transition with low specificity.

84 is a well-suited group for our purpose. First, comprehen- ${ }_{116}$ of Tetrapoda in particular and animals in general. 85 sive databases are available, including the distributional 86 ranges of most species in the group [21-23]. Second, ${ }_{87}$ the different classes within Tetrapoda possess diverse ca- ${ }_{117}$ 88 pabilities to disperse and withstand abiotic conditions. ${ }_{89}$ Therefore, we can investigate if various capabilities influ90 ence climatic niche domains, and possibly generalise the ${ }_{91}$ climatic regions to other groups. Third, there is accumu${ }_{92}$ lated evidence on the main climatic factors controlling 93 the distribution of these species, which simplifies the se94 lection of appropriate climatic variables. In particular, 95 the distribution of tetrapods is strongly determined by 96 water and energy aspects of climate [4,33-37]. Finally, ${ }_{97}$ researchers study Tetrapoda species in several disparate ${ }_{98}$ fields - from animal husbandry [38] to ecological [39] and ${ }_{99}$ evolutionary studies [40] - where a description of their 100 climatic regions can be especially useful.

101 In our classification approach, we first approximate the 102 realised niche of each species as the probability of finding 103 the species across a two-dimensional space that repre104 senting water and energy aspects of climate (Fig. 1). We 105 then use a community-detection algorithm from network 132 detected niche domains, but also observed some differ106 theory to simultaity do major do106 theory to simultaneously find portions of the climatic 134 mains with 50 or more species in the lower hierarchical ${ }_{107}$ niche space holding similar species, the niche domains, ${ }_{135}$ level is similar across Tetrapoda classes, ranging from 13 108 and the species grouped into these domains. Mapping 136 to 15. However, mammals and birds show a domain of 109 back to the Earth's surface gives for each climatic niche ${ }_{137}$ low energy, whereas reptiles present some domains across 110 domain a climatic region. We then examine the transi- 138 arid conditions, that is with elevated energy inputs and 111 tion zones and the geographical signal in the climatic re- 139 low water availability (Fig. 2). These differences seem 112 gions. The novel climatic regions confirm the existence of 140 to be related to the particular adaptations of each group ${ }_{113}$ generalised climatic constraints across life forms. There- 141 to withstand climatic conditions. Nevertheless, the clas114 fore, the climatic regions provide valuable information 142 sification of most domains was largely congruent across 115 for conservation and ecological and evolutionary studies ${ }_{143}$ classes, and hence we classified the climatic space of the 

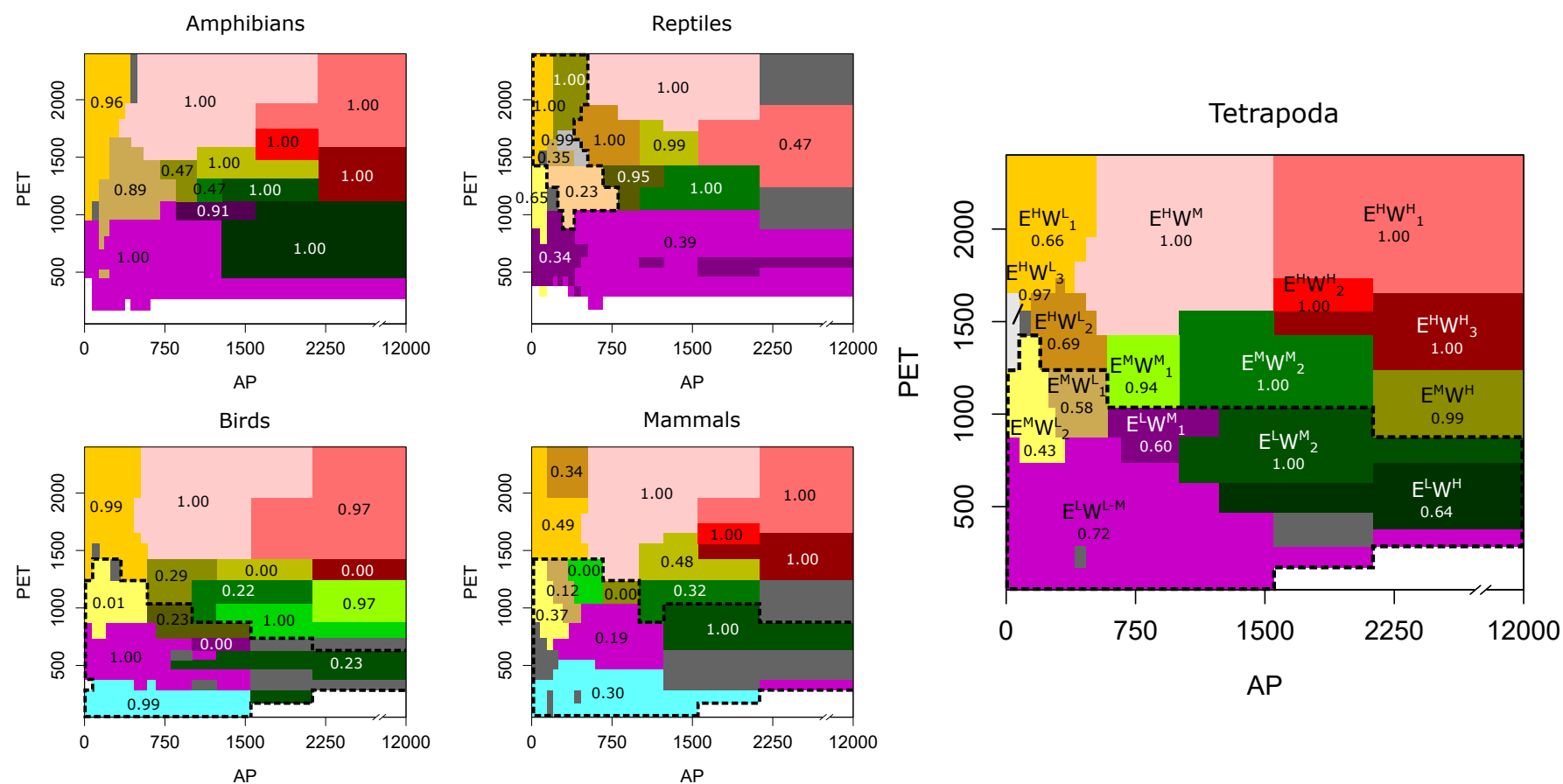

FIG. 2: Tetrapoda niche domains across the climatic space. The climatic niche domains of each group shown across a space defined by potential evapotranspiration (PET) as a surrogate of energy and annual precipitation (AP) as a surrogate of water inputs. Tetrapoda superclass domains labelled so that E and W represent energy and water, respectively, and superscripts H, M and L mean high, medium and low, respectively. Numerical subscripts differentiate domains of similar climates. Bootstrap support between 0 and 1 . The dotted line represents the domains at the highest hierarchical level. Domains formed of less than 50 species coloured in dark grey.

144 Tetrapoda superclass by using all species jointly. The 145 niche space of Tetrapoda divided into 16 main domains 146 that were similar to those of the independent classes, and 147 some of the above-explained particularities did not ap148 pear (Fig. 2).

${ }_{149}$ Since uncertainties related to the ranges of species ex150 ist, we employed a bootstrap and a significance clustering 151 procedure $[43,44]$ to asses the domain robustness (Ap152 pendix S2). While several domains were well supported, 153 we found that the niche domains corresponding to inter154 mediate energy (between approximately 1000 and 1500 ${ }_{155}$ PET units; $\mathrm{E}^{\mathrm{M}}$ climates in Fig. 2) and low to moderate ${ }_{156}$ water (up to approximately 800 m.m.l.l.; $\mathrm{W}^{\mathrm{L}}$ to $\mathrm{W}^{\mathrm{M}}$ ) 157 were among the least supported. This robustness analy158 sis shows that these niche domains are more challenging 159 to classify.

\section{Tetrapoda vs Köppen's climatic regions}

${ }_{161}$ With delineated niche domains, we studied the geo162 graphic location of their climatic conditions, the climatic 163 regions in Fig. 1 and 3, which allowed for a more pre${ }_{164}$ cise comparison between groups and Köppen's regions. ${ }_{165}$ The similarities among the regions of Tetrapoda classes 166 measured as Adjusted Mutual Information (AMI) ranged ${ }_{167}$ from 0.57 to 0.68 , with mean $\mathrm{AMI}=0.62$ (Table S1).
168 Moreover, the regions based on the niche domains of the 169 superclass Tetrapoda were mostly congruent with the re170 gions of its independent classes (mean AMI $=0.71$, rang171 ing from 0.66 to 0.77 ). Köppen's regions were more dis172 similar both to the regions of Tetrapoda $(\mathrm{AMI}=0.44)$ 173 and the ones of Tetrapoda classes (mean AMI $=0.44$, 174 ranging from 0.40 to 0.47 ).

Focusing on particular regions, we saw that climates 176 of high energy $\left(\mathrm{E}^{\mathrm{H}}\right)$ were consistent among groups and 177 Köppen's classification. Desert climates (high energy and 178 low water, $\mathrm{E}^{\mathrm{H}} \mathrm{W}^{\mathrm{L}}$, BWh and BWk according to Köppen's 179 system) were the most similar across all classifications. 180 Tropical savanna and steppe climates (high energy and 181 medium water, $\mathrm{E}^{\mathrm{H}} \mathrm{W}^{\mathrm{M}}$, Aw and BSh respectively follow182 ing Köppen) were also consistently defined, though both 183 of these Köppen regions were often classified together 184 in all groups but reptiles (Fig. 3). Similarly, Köppen's 185 tropical rainforest (Af) and tropical monsoon (Am) cli186 mates were for the most part well recovered. However, 187 we found three different tropical-humid regions, each one 188 mostly corresponding to one of the three largest masses of 189 tropical rainforests: Amazonian, African and Southeast ${ }_{190}$ Asian rainforests; $\mathrm{E}^{\mathrm{H}} \mathrm{W}^{\mathrm{H}}{ }_{1}, \mathrm{E}^{\mathrm{H}} \mathrm{W}^{\mathrm{H}}{ }_{2}$ and $\mathrm{E}^{\mathrm{H}} \mathrm{W}^{\mathrm{H}}{ }_{3}$, respec191 tively (Fig. 3). Regarding regions of low energy (conti192 nental, E, and polar climates, D, corresponding to the 193 highest hierarchical level in Köppen's system), we found 194 a slightly higher level of disagreement between Köppen's 
195 and Tetrapoda classifications (Fig. 3). Finally, temper- 24 196 ate climates (medium energy $\mathrm{E}^{\mathrm{M}}$ ) were the least congru- 240 197 ent between groups and Köppen's regions. Regions of 2 ${ }_{198}$ medium energy were at the same time the least congru199 ent among the different classifications and the least sup200 ported by the bootstrap analyses, suggesting that these 201 climates impose less restrictive conditions.

\section{Climatic transition zones}

${ }_{203}$ A complete understanding of niche domains and their 204 associated climatic regions entails exploring whether the 205 domains have hard or diffuse transitions. Climatic condi206 tions corresponding to diffuse transitions should present 207 low specificity levels to the domain where they belong ${ }_{208}$ (Fig. 1). Our network approach allows to calculate this 209 specificity by the dual classification of climatic bins and 210 species into same niche domains (Fig. 1). We com${ }_{211}$ puted the specificity of each climatic bin as the ratio ${ }^{264}$ ${ }_{212}$ between the link weights of the species classified in the ${ }_{213}$ same domain and the total link weights $[3,45]$. Then, 214 we projected these values geographically. As expected, 215 lower specificity values were in general associated with 216 the boundaries of the climatic regions (Fig. 4a and S1). ${ }_{217}$ Our results also revealed that harsh conditions, such ${ }_{218}$ as desert and continental-polar climates $\left(\mathrm{E}^{\mathrm{H}} \mathrm{W}^{\mathrm{L}}\right.$ and $\left.{ }_{219} \mathrm{E}^{\mathrm{L}} \mathrm{W}^{\mathrm{L}}\right)$, present the highest specificity levels, regardless 220 of the group (Fig. 4a and S1), reflecting the difficulties 221 to colonise these climates. Contrarily, temperate regions 222 showed the lowest levels of specificity. These regions were 223 also weakly supported in the bootstrap analysis; we found 224 that bootstrap $p$-values and mean specificity were signif225 icantly correlated (stand. Glmm. coeff. 6.21; $P<0.001$; ${ }_{226} R^{2}$ conditional $=0.29$, see Material and Methods). To227 gether with the higher variability of these regions across ${ }_{228}$ groups, this result further supports the idea that these 229 climatic conditions could impose less restrictive condi230 tions to Tetrapoda.

\section{Geographical signal in climatic regions}

${ }_{232}$ Historical and geographical processes can produce the ${ }_{233}$ detection of climates leading to specific species pools in ${ }_{234}$ some regions of the Earth but not in others. Thus,
${ }_{235}$ to explore for this geographical signal, we first com${ }_{235}$ to explore for this geographical signal, we first com-
236 pared the distribution of the climatic conditions and ${ }_{237}$ species grouped within the same niche domain. A ge${ }_{238}$ ographic mismatch between species and climate distri239 butions would point to portions of the climatic regions 240 that are defined by species occurring in other geographic ${ }_{241}$ areas. Exploring these patterns for each niche domain 242 revealed notable geographic agreement between species 243 and climatic conditions of the same domain (Figs. 4b 244 and S2-6). Nevertheless, we found some differences across 245 groups and regions. More extreme climates showed larger 246 mismatches between species and climates distributions.
${ }_{247}$ For instance, for all groups but reptiles, desert climate $248\left(\mathrm{E}^{\mathrm{H}} \mathrm{W}^{\mathrm{L}}\right)$ was mostly defined by species inhabiting Aus249 tralia and to a lesser extent by species from the Namib250 ian desert and The horn of Africa, with few or none 251 species inhabiting the Sahara desert (Figs. 4c and S2${ }_{252}$ S6). Similarly, the northern climatic regions of amphib${ }_{253}$ ians and reptiles were defined by species at lower lat254 itudes (Figs. S2-3). Approaching the geographical sig255 nal more quantitatively (see Material and Methods), we 256 found a stronger signal for the worse dispersers amphib257 ians and reptiles than for mammals and birds (Fig. 4d), 258 suggesting that dispersal capabilities can contribute to 259 the geographical signal in the niche domains. Finally, 260 the Tetrapoda superclass showed the lowest geograph261 ical signal, which suggests that, beyond dispersion, an 262 increased evolutionary time can reduce the geographical 263 signal.

\section{DISCUSSION}

${ }_{265}$ We detected 16 climatic regions governing the distri266 bution of Tetrapoda. Despite the substantial physiolog${ }_{267}$ ical and functional differences among the groups, most ${ }_{268}$ of their niche domains and climatic regions are consis269 tent. Some of these climatic regions resemble Köppen's 270 regions, which supports the idea that general climatic ${ }_{271}$ constraints organise the distribution of life on Earth.

${ }_{272}$ While we found a high general congruence across ${ }_{273}$ groups, some niche domains and climatic regions were 274 more consistent than others. In general, more extreme 275 climates, such as arid or low-energy continental areas, 276 were well defined in all groups. These climates also 277 presented high levels of specificity, showing that species 278 adapted to other climates have more difficulties to with279 stand these conditions. Both of these results suggest that 280 extreme climates impose strong adaptive barriers [46, 47], 281 even across distinctive evolutionary lineages.

282 Contrarily, milder climatic conditions, especially tem283 perate climates, showed the lowest specificity, statisti284 cal support, and congruence across groups. These cli285 mates are more difficult to classify due to the overlap 286 in the climatic space of species pools with different cli287 matic optima. Two complementary reasons can explain 288 this ambiguity: First, while we used two variables widely 289 recognised to shape Tetrapoda distributions, alternative 290 variables, such as seasonal changes of energy and precip291 itation [48], may also influence species inhabiting tem292 perate regions. Including these variables might help to 293 further separate temperate species pools across the cli294 matic space. Second, the climatic conditions of these do295 mains may not prevent the colonisation of species with 296 other realised optima or preferences, which would gen${ }_{297}$ erate the observed overlap in the climatic space across 298 milder conditions. Questions remain about the relative 299 contribution of each factor.

We also found some domains that were well supported 301 but unique for each group. These differences between 
bioRxiv preprint doi: https://doi.org/10.1101/851030; this version posted November 22, 2019. The copyright holder for this preprint (which was not certified by peer review) is the author/funder, who has granted bioRxiv a license to display the preprint in perpetuity. It is made available under aCC-BY-NC 4.0 International license.

$\mathbf{a}$
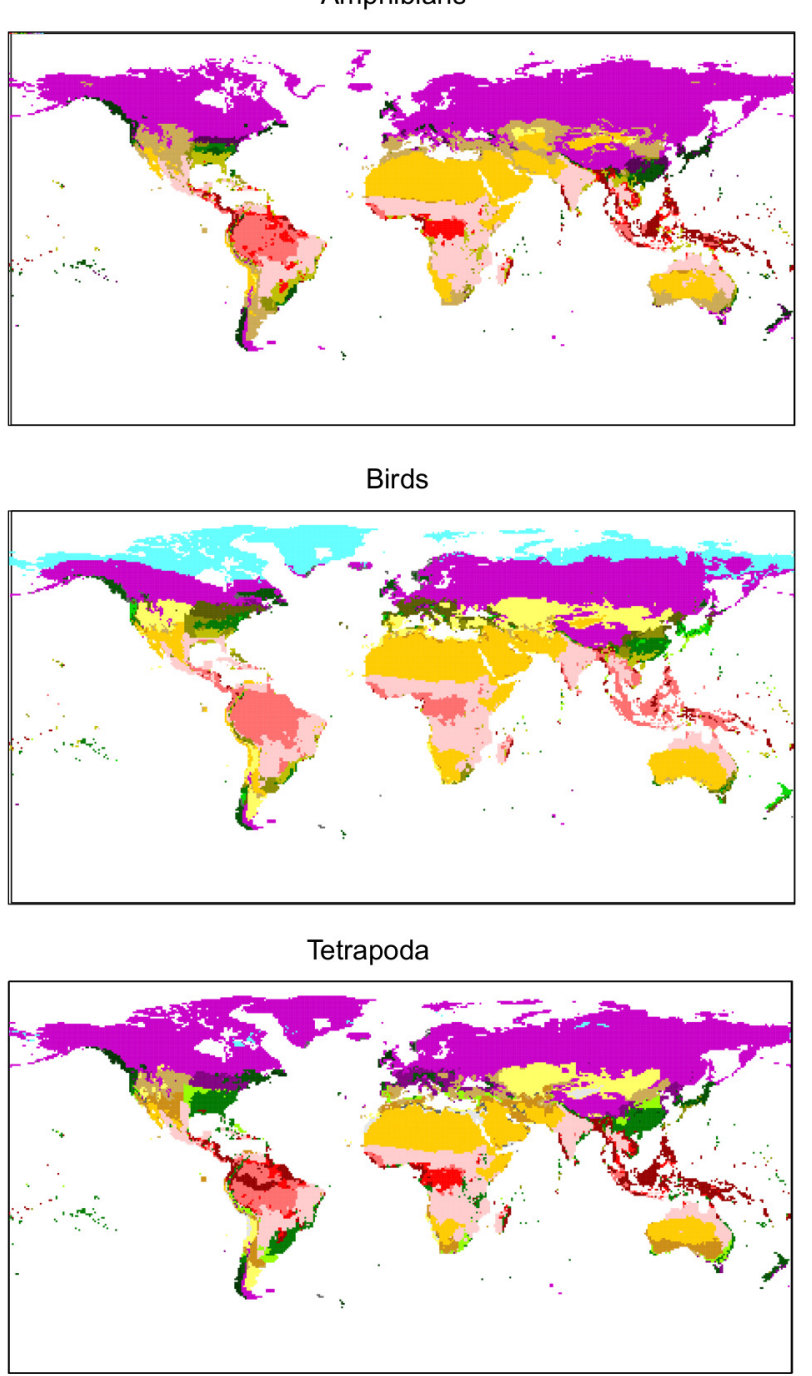

Reptiles
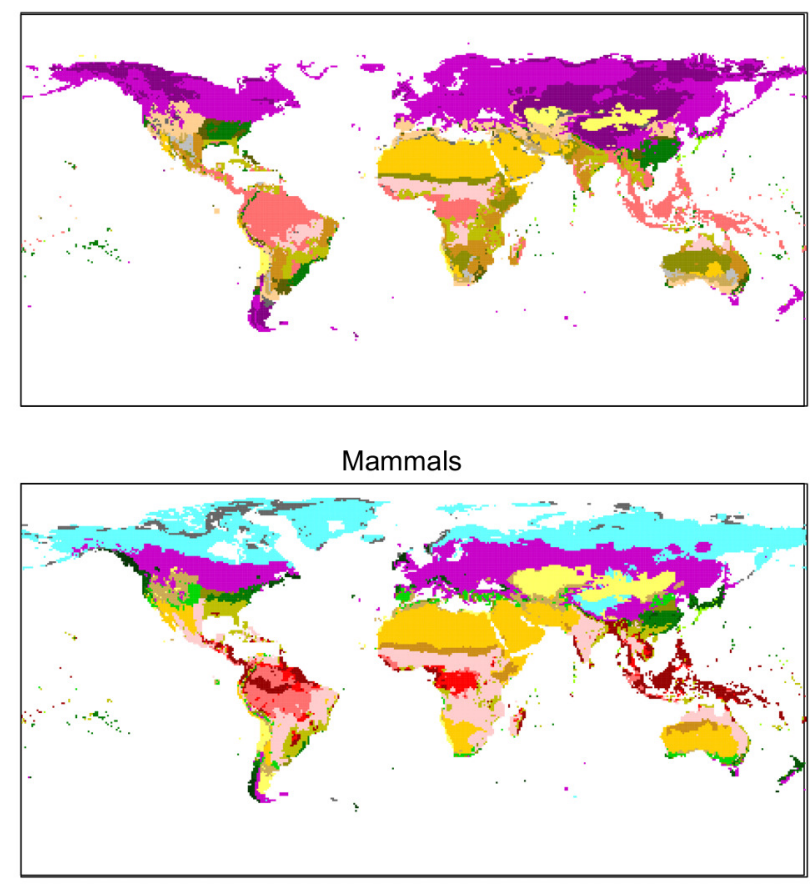

Köppen

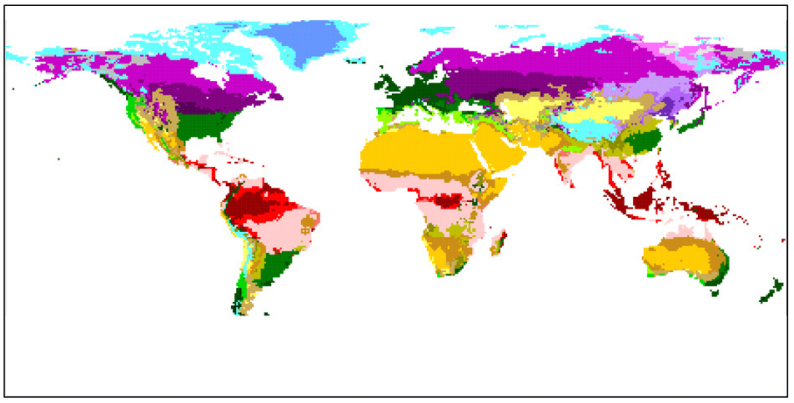

b

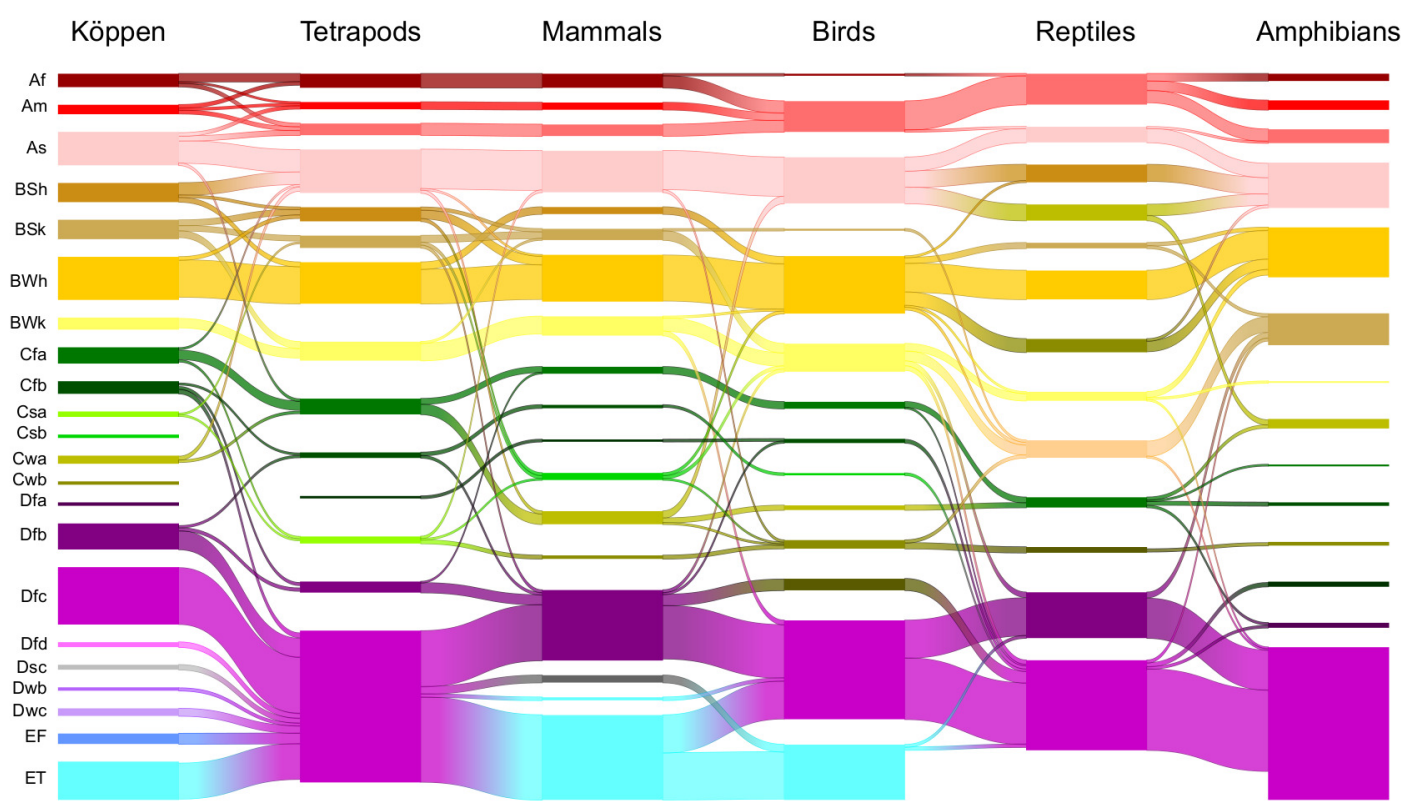

FIG. 3: Tetrapoda groups and Köppen's climatic regions are largely congruent. a Geographic location of Tetrapoda niche domains and Köppen's climatic regions. b Alluvial diagram showing the similarities among the climatic regions. Colours according to Fig. 2. 
302 groups seem to relate to the particular physiological 303 adaptations of each group. For instance, homeothermic 304 birds and mammals defined a region of low energy, con305 sistent with Köppen's polar climates, that reptiles and 306 amphibians lacked. Similarly, reptiles, a group holding 307 several species adapted to arid environments [36], de308 fined some regions of low precipitation and high PET. ${ }_{309}$ Hence, despite the high similarities among groups, our 310 results stress that caution is needed when generalising 311 the climatic regions to other groups of organisms.

312 Beyond niche domains, our results also show differ313 ences in the geographical signal across groups. That 314 amphibians - species with the lowest dispersal capac315 ity - showed the highest geographical signal suggests 316 that dispersal processes play an essential role: worse dis${ }_{317}$ perser species have more difficulties tracking their pre318 ferred climates [49], limiting the colonisation of disjoint 319 areas with similar climates. Moreover, the Tetrapoda 320 superclass shows the lowest geographical signal, which 321 suggests that a increased evolutionary time can reduce 322 this effect. Thus, evolutionary history - through the ap${ }_{323}$ pearance of convergent adaptations to similar climates in 324 different geographic regions[50] - may also influence the 325 geographical signal in niche domains. In any case, the 326 ultimate causes and consequences of this signal require 327 further attention. Why are some amphibians able to in328 habit arid conditions in the Australian desert but not 329 in the Sahara desert (Fig. S2)? Why can some reptiles 330 withstand cold climates in and around the Himalayan 331 mountains but not at the high latitudes of the northern 332 hemisphere (Fig. S3)? These are some of the emerging ${ }_{333}$ fundamental questions whose answers require historical 334 biogeographical and evolutionary approaches.

${ }_{335}$ Our results bring us closer to a definition of climatic 336 regions that represent active factors for the organisation ${ }_{337}$ and evolution of life. Nevertheless, it would be interesting 338 to improve some aspects in future studies. First, while 339 we used a large number of species (about 26,000), they 340 are taxonomically biased and only represent a small frac341 tion of the terrestrial organisms. Similarly, we used two 342 climatic variables widely known to affect the distribution 343 and diversity patterns of animals and plants in general $344[4,33]$, but other climatic variables might refine some of 345 the least supported regions. Finally, our domains repre346 sent portions of the realised climatic niche space, and this ${ }_{347}$ space may be influenced by historical, geographical, and 348 biotic factors beyond pure climate $[3,27,51]$. Although 349 the geographical signal was rather low, identifying po350 tential niches may also improve the accuracy of climatic 351 regions. At the current pace of biological data accumula352 tion and computational development, it is reasonable to 353 expect that some of these limitations will soon be over${ }_{354}$ come. Meanwhile, the considerable congruence of several 355 climatic regions across the studied groups and Köppen's 356 system provides confidence in their robustness. Hence, it 357 is likely that using more and better data would not pro358 duce regions substantially different from those presented 359 here.

Regardless of how generalisable the results are, the 361 niche domains and their associated species pools and cli362 matic regions can be used as a basis for ecological and 363 evolutionary studies, as well as for conservation planning 364 concerning Tetrapoda. Some of the many questions that 365 the results reported here (data available in Appendix S3) 366 can help to answer include : Are all the climatic regions 367 similarly conserved and/or protected? Do the species 368 forming each niche domain differ functionally or phy369 logenetically? Is the adaptation to niche domains evo370 lutionary constrained? Do diversification, extinction or 371 speciation rates differ among the species associated with 372 different domains?

In conclusion, our data-driven climate classification re374 veals major climatic boundaries organising the distribu375 tion of life on Earth. Questions remain regarding the 376 mechanism underlying differences between groups in the 377 climatic regions and the geographical signal. Neverthe378 less, the regions that are consistent across groups can 379 help answer questions in a diverse array of fields, includ380 ing climatology, geography, ecology, evolution and con381 servation.

\section{2}

MATERIAL AND METHODS

$383 \quad$ Data

We obtained the distribution ranges of mammals and 385 amphibians from The IUCN Red List of Threatened 386 Species [21], of birds from Bird species distribution maps 387 of the world [22] and of reptiles from ref. [23]. We in388 cluded only the native range of terrestrial species in the 389 analyses in all instances. In the case of birds, we only 390 used the breeding ranges. Moreover, since there is a 391 higher uncertainty when determining the realised niches 392 of narrow-ranging species [52], we removed the species 393 whose ranges were less than 5 grid cells of 0.5 degrees. 394 After this cleaning of the data, we used 3657 amphibians, 3957204 reptiles, 4574 mammals and 10684 birds, for a total 396 of 26119 Tetrapoda species.

We approximated the species' Grinnellian niches[51] 398 with two climatic variables that represent energy and wa399 ter inputs. While we could have used several other vari400 ables, we chose energy and water since they best explain 401 climatic effects on species distributions [4]. As surrogates ${ }_{402}$ of energy and water inputs, we used mean annual poten403 tial evapotranspiration (PET) and annual precipitation, 404 respectively. Both variables have been shown to be im${ }_{405}$ portant factors for Tetrapoda species distributions [33$\left.{ }_{406} 35\right]$. Moreover, they have also been used in previous cli407 mate classifications [18] and are regularly used to derive 408 other drivers of species distributions such as the UNEP 409 aridity index $[53,54]$. We obtained PET from ref. [25] 410 and annual precipitation from ref. [24], both at a $0.08^{\circ}$ ${ }_{411}$ resolution. Finally, we obtained Köppen's climatic re${ }^{412}$ gions from refs. $[9,55]$. 


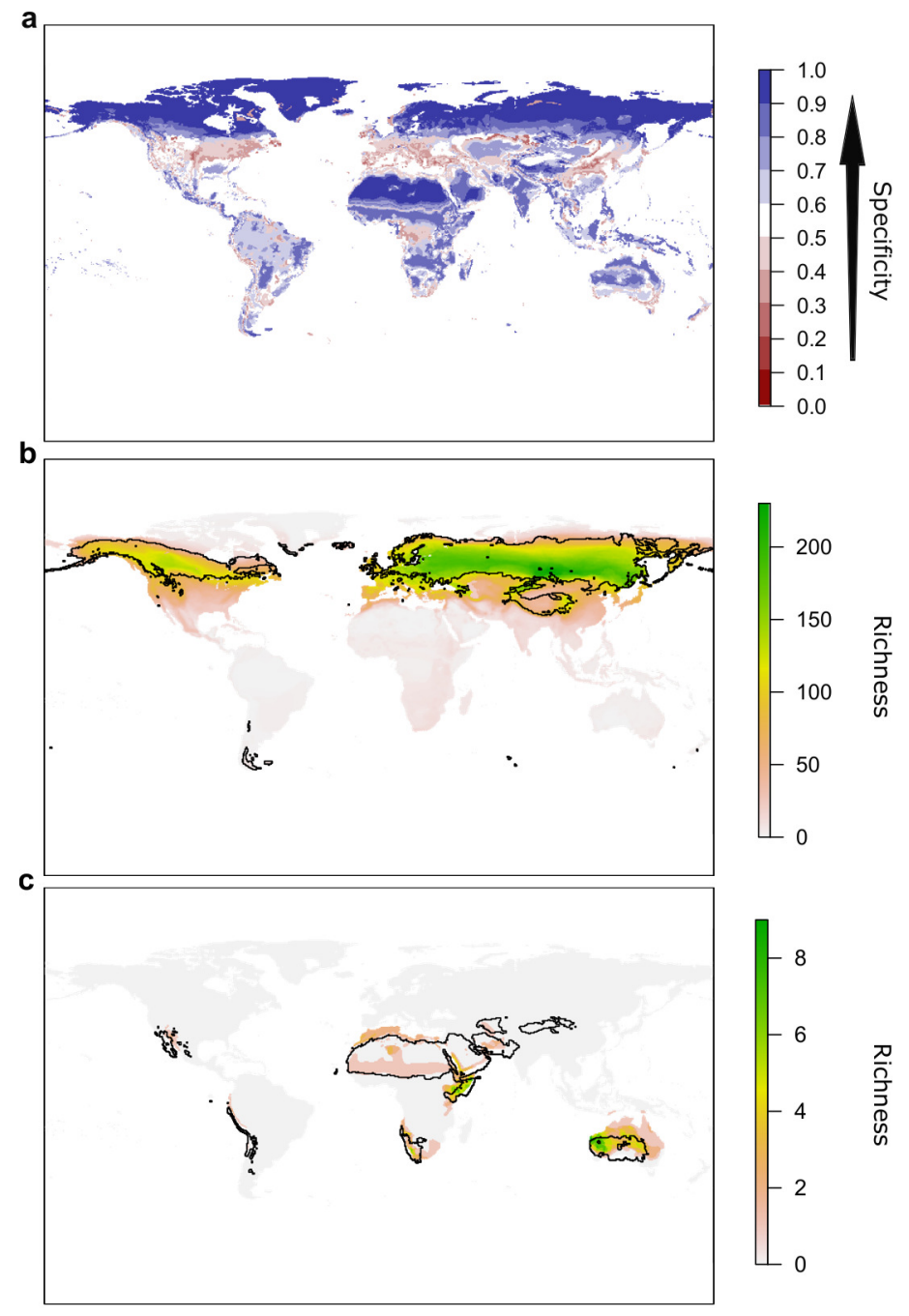

d

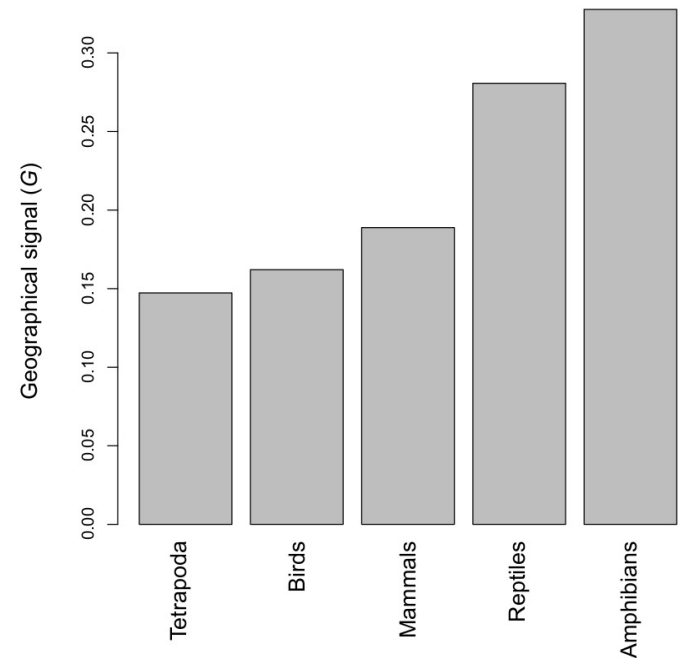

FIG. 4: The geographic location of climatic domains and their associated species provide insights into the mechanism underlying the climatic regions. a Geographic projection of the specificity of climatic bins to their niche domain. b An example showing a bird's niche domain with a low geographical signal. The distribution of the climatic conditions (black line) and the species (coloured richness values) belonging to the same niche domain were mostly congruent. c An example of an amphibian's niche domain showing a high geographical signal, reflected in a substantial mismatch between the distribution of climatic conditions and species belonging to the same domain.

d A quantitative approximation of the geographical signal, ranging between 0 and 1 , for the different taxonomic groups (see Materials and Methods). 
${ }_{414}$ We characterised the realised climatic niche of each 415 species using an approach similar to the one proposed in ${ }_{416}$ ref.[26]. We divided the climatic space formed by PET ${ }_{417}$ and annual precipitation into bins and calculated the pro${ }_{418}$ portion of occurrences a given species has in each climatic ${ }_{419}$ bin. Both the shape of the divisions and the number of ${ }_{420}$ divisions of each climatic axis affect the result. For in${ }_{421}$ stance, dividing the axis into regular intervals can destroy ${ }_{422}$ critical information if the climatic values more restrictive ${ }_{423}$ are skewed toward any extreme of the distribution or if ${ }_{424}$ the climatic values are represented non-uniformly across 425 the globe (as for annual precipitation, Fig. S7). Also ${ }_{426}$ dividing the space into too few intervals destroys infor${ }_{427}$ mation, whereas using too many divisions can generate ${ }_{428}$ niche domains with only a few species. To overcome the 429 first issue, we divided the axes in quantiles based on the 430 distribution of climatic values across the Earth. By do${ }_{431}$ ing so, we obtained an almost uniformly divided PET ${ }_{432}$ axis (Fig. S7). Contrarily, the number of divisions of the ${ }_{433}$ annual precipitation axis was skewed towards low values, ${ }_{434}$ which resulted in a higher resolution over the presumably 435 more relevant low-precipitation conditions (Fig. S7). To 436 solve the second issue, we selected the lowest number of ${ }_{437}$ divisions that maximised the gain in information (see Ap${ }_{438}$ pendix S1). The optimal number of axis divisions was 17 439 in all cases but amphibians, where it was 18 (Fig. S8).

${ }_{440}$ Next we accounted for potential commission errors, ${ }_{441}$ which may affect the different climates a species expe${ }_{442}$ riences. Specifically, range maps can overestimate the ${ }^{495}$ ${ }_{443}$ area occupied by a species, which directly influences the ${ }_{444}$ niche characterisation [56]. Extracting the climatic val${ }_{445}$ ues that a species range covers from a high-resolution ${ }_{446}$ climatic raster (such as $0.08^{\circ}$ ) may reduce commission ${ }_{447}$ errors at the borders of the species range, but increases 448 this error otherwise. Extracting climatic values from a 500 ${ }_{449}$ coarser raster can reduce the influence of commission er450 rors over the areas inside of a range but increases them ${ }_{451}$ over the borders. To alleviate the effects of these poten${ }_{452}$ tial errors, we first extracted the climatic values from the 504 ) ${ }_{453}$ high-resolution rasters $\left(0.08^{\circ}\right)$. Then, we computed the ${ }^{50}$ ${ }_{454}$ average climatic values among selected raster pixels lo- 506 455 cated within cells of 0.5 degrees. In this way, we reduced ${ }_{456}$ the effects of commission errors both at the borders of ${ }_{457}$ and inside species ranges. Moreover, we also conducted ${ }_{458}$ a bootstrap significance test that takes uncertainty of 459 species ranges into account (see below).

\section{Niche domains and climatic regions identification}

\section{${ }_{461}$ We employed a network community detection ap-} ${ }_{462}$ proach to identify the niche domains and the species ${ }_{463}$ mainly associated with them. For each group of species, ${ }_{464}$ we first generated a weighted bipartite network where 465 species and climatic bins formed the disjoint sets of 466 nodes, and the proportion of occurrences of species in
467 intervals of the climatic values corresponding to the cli468 matic bins formed the weighted links. We then used 469 the hierarchical version of the community detection al470 gorithm known as Infomap $[41,42]$ to identify the niche 471 domains. We ran the algorithm 1000 times, selecting the 472 network partition with the best quality.

To consider the uncertainty associated with both the 474 species ranges and the community detection, we con475 ducted a bootstrap analysis. For each species, we resam476 pled with replacement from the distribution of climatic 477 values within species ranges at a resolution of $0.08^{\circ}$. We 478 averaged climatic values laying within $0.5^{\circ}$ cells and cal479 culated the proportion of occurrences in each climatic 480 bin. With resampled data from all species, we generated 481 a bootstrapped network and ran Infomap 1000 times us482 ing this network. Given the high computational cost of 483 this analysis, we only generated 100 bootstrap networks. ${ }_{484}$ We followed the approach proposed in ref. [44] to calcu485 late the support of the niche domains. For each identified 486 domain, we calculated the proportion of bootstrap net487 works with a domain more similar than Jaccard index $4880.5[44]$.

489 With obtained niche domains, we detected the climatic 490 regions by identifying areas across the Earth's surface 491 that hold the climatic conditions grouped within each 492 niche domain. Finally, to compare climatic regions across 493 Tetrapoda groups and with Köppe's classification, we cal494 culated the adjusted mutual information (AMI) [57].

\section{Climatic transition zones}

The joint classification of climatic bins into domains 497 and the species most associated to them allowed us to 498 calculate the specificity of the bins to the domain where 499 they belong, which indicates zones of transitions between 500 domains (Fig. 1). That is, a bin acting as a transition 501 between two domains should contain species from both 502 domain and, therefore, a low specificity to the domain 503 where it is classified $[3,45]$. To consider the link weights, 504 we calculated this specificity $S_{i}^{D}$ of a climatic bin $i$ in 505 domain $D$ as the sum of link weights $w_{i, j}$ of the species $j$ 506 present in the bin and also belonging to the same domain 507 as the bin, divided by the sum of link weights of all the 508 species present in the bin, such that

$$
S_{i}^{D}=\frac{\sum_{j \in D} w_{i, j}}{\sum_{j} w_{i, j}} .
$$

509 This index is 1 when the bin has only species of the 510 same domain as the bin, and tends to 0 otherwise. We 511 then projected these specificity values into the geographic 512 space by assigning these values to the geographical raster 513 cells $q$ that hold the climatic conditions represented by 514 the bins, thus obtaining the projected specificity $S_{q}^{P}$. Fi515 nally, we explored the relationship between average $S^{D}$ 516 and bootstrap support. We fitted a logistic GLMM of 517 bootstrap $p$-values as function of mean $S^{D}$ as fixed term 
518 and the taxonomic group as a random intercept term. 549 species.

${ }_{519}$ GLMM was conducted using the lme4 [58] package in ${ }_{550}$ Using Eq. 1, we calculated the actual specificity of a ${ }_{520} \mathrm{R}[59]$. 551 geographical raster cell $q$, whose corresponding climatic ${ }_{552}$ bin $i$ is in domain $D$, as the ratio between the link weights 553 of species in raster cell $q$ that belong to its associated 554 domain and the total link weights of species in $q$,

522 To investigate the geographical signal, we first ob${ }_{523}$ served the match between the geographic location of the ${ }_{524}$ species and the climatic conditions associated with the ${ }_{525}$ corresponding niche domain. Then, we quantified the 526 geographical signal by comparing the geographically pro${ }_{527}$ jected specificity $S^{P}$ with a measure of specificity based 528 on the actual pool of species co-occurring geographically. ${ }_{529}$ That is, the specificity of a climatic bin $S^{D}$ is based on 530 the species that co-occur in the climatic space and then ${ }_{531}$ it is projected geographically to obtain $S^{P}$ (see above). ${ }_{532}$ Hence, $S^{P}$ does not considered the actual pool of species ${ }_{533}$ co-occurring in the geographic space. In case of a large ${ }_{534}$ geographical signal, we would expect large differences be- 560 where $N$ is the total number of raster cells. This index ${ }_{535}$ tween the species co-occurring in the climatic and geo- 561 is 0 when there is no geographical signal and tends to 1 536 graphic spaces. For instance, the geographic mismatch 562 for high signals.

${ }_{537}$ between species and climates belonging to the same do- ${ }_{563}$ Competing interests. The authors declare no com${ }_{538}$ main is produced by species co-occurring in a given por- 564 peting interests. Author contribution. J.C. and M.N. 539 tion of the climatic space but not in all geographical areas 565 conceived the ideas with inputs from all authors; J.C. ${ }_{540}$ with the climate represented in such portion of the cli- 566 analysed the data with assistance from all authors; J.C. ${ }_{541}$ matic space. In this sense, in case of geographical signal 567 wrote the manuscript in collaboration with all authors. ${ }_{542}$ we would expect differences between the projected speci- 568 Acknowledgements. We are thankful to Andrea Br${ }_{543}$ ficity $S^{P}$ and a value of specificity based on the species 569 iega and Miguel Á. Rodríguez for discussion on early ${ }_{544}$ pool occurring in given geographical areas, for short the 570 ideas. We are very grateful to Fernanda Alves-Martins, ${ }_{545}$ actual specificity $S^{A}$. A higher actual specificity than the ${ }_{571}$ Rafaél Molina-Venegas, Cristina Roquillo and Rubén ${ }_{546}$ projected indicates areas that host most of the species 572 Bernardo-Madrid for critical reviews. J.C. is supported ${ }_{547}$ associated with a niche domain, while the opposite in- 573 by the Carl Tryggers Foundation for Scientific Research 548 dicates areas not, or only scarcely, colonised by these 574 (CTS 16:384).
[1] F. I. Woodward, Climate and plant distribution (Cam- 597 bridge University Press, 1987).

[2] A. A. Hoffmann and P. A. Parsons, Extreme environmen- 50 tal change and evolution (Cambridge University Press, 600 1997).

[3] J. Calatayud, M. A. Rodriguez, R. Molina-Venegas, M. Leo, J. L. Horreo, and J. Hortal, Proceedings of the 603 Royal Society B 286, 20190291 (2019).

[4] B. A. Hawkins, R. Field, H. V. Cornell, D. J. Currie, J.-F. 605 Guégan, D. M. Kaufman, J. T. Kerr, G. G. Mittelbach, 606 T. Oberdorff, E. M. O'Brien, et al., Ecology 84, 3105 (2003).

[5] H. Kreft and W. Jetz, Proceedings of the National Academy of Sciences 104, 5925 (2007).

[6] R. H. Whittaker, The Botanical Review 28, 1 (1962).

[7] M. Sanderson, Bulletin of the American Meteorological Society 80, 669 (1999).

[8] J. E. Oliver, Physical Geography 12, 231 (1991).

[9] M. Kottek, J. Grieser, C. Beck, B. Rudolf, and F. Rubel, Meteorologische Zeitschrift 15, 259 (2006).

[10] C. W. Thornthwaite, Geographical Review 33, 233 (1943).
${ }_{597}$ [11] R. V. Rohli, T. A. Joyner, S. J. Reynolds, and T. J. Ballinger, Physical Geography 36, 158 (2015).

[12] J. Spinoni, J. Vogt, G. Naumann, H. Carrao, and P. Barbosa, International Journal of Climatology 35, 2210 (2015).

[13] P. Gentine, P. D'Odorico, B. R. Lintner, G. Sivandran, and G. Salvucci, Geophysical Research Letters 39 (2012).

004 [14] P. C. Tobin, J. M. Kean, D. M. Suckling, D. G. McCullough, D. A. Herms, and L. D. Stringer, Biological Invasions 16, 401 (2014).

$715]$ R. A. Garcia, M. Cabeza, C. Rahbek, and M. B. Araújo, Science 344, 1247579 (2014).

[16] G. T. Trewartha, An introduction to climate (McGRAW${ }_{610}$ HILL BOOK COMPANY, INC. NEW YORK ${ }_{611}$ TORONTO LONDON, 1954).

612 [17] L. R. Holdridge, Science 105, 367 (1947).

${ }_{613}$ [18] C. W. Thornthwaite, An approach toward a rational clas${ }_{614}$ sification of climate, Vol. 66 (LWW, 1948).

615 [19] P. Netzel and T. Stepinski, Journal of Climate 29, 3387 ${ }_{616}$ (2016).

${ }_{617}$ [20] GBIF: The Global Biodiversity Information Facility, 618 What is GBIF? (2019). 
${ }_{619}[21]$ IUCN, The IUCN Red List of Threatened Species (2015). 664

620 [22] BirdLife, Bird species distribution maps of the world 665 $621 \quad(2015)$.

2023] U. Roll, A. Feldman, M. Novosolov, A. Allison, A. M. 600 [

${ }_{623}$ Bauer, R. Bernard, M. Böhm, F. Castro-Herrera, 668

624 L. Chirio, B. Collen, et al., Nature Ecology \& Evolution 669

$625 \quad \mathbf{1}, 1677(2017)$.

[24] S. E. Fick and R. J. Hijmans, International journal of 671

${ }_{627} \quad$ climatology $\mathbf{3 7}, 4302(2017)$.

628 [25] A. Trabucco and R. J. Zomer, CGIAR Consortium for

${ }_{629}$ Spatial Information (2009).

630 [26] O. Broennimann, M. C. Fitzpatrick, P. B. Pearman, 675

${ }_{631}$ B. Petitpierre, L. Pellissier, N. G. Yoccoz, W. Thuiller,

${ }_{632}$ M.-J. Fortin, C. Randin, N. E. Zimmermann, et al., 677

${ }_{633}$ Global ecology and biogeography 21, 481 (2012).

634 [27] D. L. Warren, M Cardillo, D. F Rosauer, and D. I.

${ }_{635}$ Bolnick, Trends in Ecology \& Evolution 29, 572 (2014).

636 [28] J. Calatayud, J. L. Hórreo, J. Madrigal-González, A. Mi- 681

${ }_{637}$ geon, M. Á. Rodríguez, S. Magalhães, and J. Hortal, ${ }_{682}$

${ }_{638}$ Proceedings of the National Academy of Sciences 113, 683

$6399840(2016)$.

640 [29] R. E. Ricklefs, Science 235, 167 (1987).

641 [30] R. C. Flohr, C. J. Blom, P. B. Rainey, and H. J. Beau-

${ }_{642}$ mont, Proceedings of the National Academy of Sciences ${ }^{687}$

${ }_{643} \mathbf{1 1 0}, 20663(2013)$.

644 [31] H. Tuomisto, K. Ruokolainen, and M. Yli-Halla, Science

645 299, 241 (2003).

646 [32] E. F. Connor and E. D. McCoy, The American Naturalist

647 113, 791 (1979).

648 [33] D. J. Currie, The American Naturalist 137, 27 (1991).

649 [34] M. W. Tingley, W. B. Monahan, S. R. Beissinger, and 69

${ }_{650}$ C. Moritz, Proceedings of the National Academy of Sci- 695

651 ences 106, 19637 (2009).

652 [35] S. F. Gouveia, J. Hortal, M. Tejedo, H. Duarte, F. A. ${ }^{697}$

${ }_{653}$ Cassemiro, C. A. Navas, and J. A. F. Diniz-Filho, Global 690

${ }_{654} \quad$ Ecology and Biogeography 23, 446 (2014). 699

655 [36] M. R. Pie, L. L. Campos, A. L. Meyer, and A. Duran, 700

${ }_{656}$ Proceedings of the Royal Society B: Biological Sciences 701

$657 \quad 284,20170268$ (2017)

${ }_{658}[37]$ N. Cooper, R. P. Freckleton, and W. Jetz, Proceedings 703

${ }_{659}$ of the Royal Society B: Biological Sciences 278, 2384

660 (2011).

${ }_{661}^{6}[38]$ J. Abecia, J. Máñez, A. Macias, A. Laviña, C. Palacios, 706

${ }_{662}$ et al., J Anim Behav Biometeorol 5, 124 (2017).

${ }_{663}$ [39] D. Englert Duursma, R. V. Gallagher, and S. C. Griffith,
Ecography 42, 535 (2019).

[40] J. Rolland, F. L. Condamine, F. Jiguet, and H. Morlon, PLoS Biology 12, e1001775 (2014).

[41] M. Rosvall and C. T. Bergstrom, Proceedings of the National Academy of Sciences 105, 1118 (2008).

[42] M. Rosvall and C. T. Bergstrom, PloS one 6, e18209 (2011).

[43] M. Rosvall and C. T. Bergstrom, PloS one 5, e8694 (2010).

[44] J. Calatayud, R. Bernardo-Madrid, M. Neuman, A. Rojas, and M. Rosvall, arXiv preprint arXiv:1905.11230 (2019).

[45] R. Bernardo-Madrid, J. Calatayud, M. González-Suarez, M. Rosvall, P. M. Lucas, M. Rueda, A. Antonelli, and E. Revilla, Ecology Letters (2019).

[46] B. J. Butterfield, Oikos 124, 1374 (2015).

[47] M. W. Cadotte and C. M. Tucker, Trends in ecology \& evolution 32, 429 (2017).

[48] W. Köppen and R. Geiger, Handbuch der klimatologie, Vol. 1 (Gebrüder Borntraeger Berlin, 1930).

[49] M. B. Araújo and R. G. Pearson, Ecography 28, 693 (2005).

[50] F. Mazel, R. O. Wüest, M. Gueguen, J. Renaud, G. F. Ficetola, S. Lavergne, and W. Thuiller, Current Biology 27, 1369 (2017).

[51] J. Soberón, Ecology letters 10, 1115 (2007).

690 [52] A. Lehmann, J. Leathwick, and J. M. Overton, Biodiversity \& Conservation 11, 2217 (2002).

2 [53] N. M. UNEP and D. Thomas, Edward Arnold, London , 15 (1992).

454 A. Fuller, D. Mitchell, S. K. Maloney, and R. S. Hetem, Climate Change Responses 3, 10 (2016).

${ }_{6}^{6}$ [55] F. Rubel, K. Brugger, K. Haslinger, and I. Auer, Meteorologische Zeitschrift 26, 115 (2017).

[56] C. Rondinini, K. A. Wilson, L. Boitani, H. Grantham, and H. P. Possingham, Ecology letters 9, 1136 (2006).

[57] N. X. Vinh, J. Epps, and J. Bailey, Journal of Machine Learning Research 11, 2837 (2010).

[58] D. Bates, M. Mächler, B. Bolker, and S. Walker, Journal of Statistical Software 67, 1 (2015).

[59] R Core Team, $R: A$ Language and Environment for Statistical Computing, R Foundation for Statistical Computing, Vienna, Austria (2018). 